\title{
Emerging Animal Parasitic Diseases: A Global Overview and Appropriate Strategies for their Monitoring and Surveillance in Nigeria
}

\author{
Ngongeh L. Atehmengo ${ }^{1 *}$ and Chiejina S. Nnagbo ${ }^{2}$ \\ ${ }^{1}$ Department of Veterinary Microbiology and Parasitology, College of Veterinary Medicine, Michael Okpara University \\ of Agriculture, Umudike, Abia State, Nigeria \\ ${ }^{2}$ Department of Veterinary Parasitology and Entomology, Faculty of Veterinary Medicine, University of Nigeria, Nsukka
}

\begin{abstract}
Emerging animal parasitic diseases are reviewed and appropriate strategies for efficient monitoring and surveillance in Nigeria are outlined. Animal and human parasitic infections are distinguished. Emerging diseases have been described as those diseases that are being recognised for the first time or diseases that are already recorded but their frequency and/or geographic range is being increased tremendously. Emergence of new diseases may be due to a number of factors such as the spread of a new infectious agent, recognition of an infection that has been in existence but undiagnosed, or when it is realised that an established disease has an infectious origin. The terms could also be used to describe the resurgence of a known infection after its incidence had been known to have declined. Emerging infections are compounding the control of infectious diseases and huge resources are being channeled to alleviate the rising challenge. The diseases are numerous and include helminth, protozoal / rickettsial and entomological. A list of parasitic emerging diseases in Nigeria is included. Globally occurring emerging parasitic diseases are also outlined. Emerging and reemerging infections can be brought about by many factors including climate change and global warming, changes in biodiversity, population mobility, movement of animals, globalisation of commerce/trade and food supply, social and cultural factors such as food eating habits, religious beliefs, farming practices, trade of infected healthy animals, reduction in the available land for animals, immune-suppressed host and host density and misuse or over use of some drugs leading to drug resistance.
\end{abstract}

Keywords: Animal parasitic diseases, causes, control strategies, emerging, re-emerging.

\section{INTRODUCTION}

\subsection{Definition of Emerging and Re-Emerging Animal Parasitic Diseases}

Emerging infectious diseases (EID) are those diseases that have been newly appeared or that have existed in the past but are now rapidly increasing in frequency, geographical range, or both. For instance, the United State Institute of Medicine has defined emerging infections as those whose incidences in humans have increased within the past two decades or threatens to increase in the near future. Emergence could be due to a new agent being widely distributed or the diagnosis of a pathogen that has been present and causing disease without detection. It could also be due to discovery that an existing pathogen is actually infectious. Emergence has also been used to describe the reappearance of a known infection after a decline in incidences [1]. World health organisation (WHO)/Food and agricultural organisation (FAO)/ Office international des

*Address correspondence to this author at the Department of Veterinary Microbiology and Parasitology, College of Veterinary Medicine, Michael Okpara University of Agriculture, Umudike, Abia State, Nigeria;

Tel: +2348064645442; E-mail: mapalah@ gmail.com epizooties (OIE) joint consultation on emerging zoonotic diseases in May, 2004 defined an emerging zoonosis as a zoonosis that is newly recognised or newly evolved or that has occurred previously but shows an increase in incidence or expansion in geographical, host or vector range [2]. Emerging infectious diseases are said to be dominated by zoonoses with majority of them originating from wildlife [3].

Parasitic diseases can be broadly divided into two classes, namely animal health parasitic diseases which affect animals and human health parasitic diseases which affect humans. However, there is no clear distinction between them in the case of the zoonotic parasites which have the potential to infect both man and animals, thereby affecting the health of both classes. A good example of a zoonosis is echinoccocosis. The infection in the dog is caused by adult Echinococcus granulosus and is usually not very harmful to the dog. In humans, however, echinoccocosis is caused by the metacestode of E. granulosus known as hydatid cyst and this disease in humans is preferably termed hydatidosis. Echinococcus granulosus has an indirect life cycle with ruminants serving as the main intermediate hosts. However, man like the intermediate host of E. granulosus can also acquire the infection by ingesting viable eggs of E. granulosus. The ingested eggs develop in man into 
hydadtid cysts and lodge in organs such as eyes, liver, lungs and the central nervous system causing a fatal disease condition termed hydatidosis. A second example is Taenia solium [4] which is an infection in pigs due to the larval stage of T. solium known as Cysticercus cellulosae. Pigs are the usual intermediate hosts of $T$. solium and acquire the infection by ingestion of viable eggs of $T$. solium. Man can also be infected by ingestion of the eggs of the parasite and the eggs develop into cysts in some organs such as brain and eyes. The human cysticercosis is increasingly being reported in some countries like Latin America. Cysticercosis is common in Nigeria certainly in part due to the increasing epidemiological factors such as poor environmental hygiene coupled with poor water supply.

\subsection{Significance of Emerging and Re-emerging Animal Parasitic Diseases}

Emerging zoonotic diseases hamper both human and animal health and also cause economic loss. Emergence of drug resistant pathogens poses many challenges to governments of either channelling a number of resources for control the infections or lose the animals or humans.

\subsection{Overview of Emerging and Re-emerging Animal Parasitic Diseases}

The ecosystem is increasingly and continually being put into turmoil by human acts such as deforestation and global warming with the resultant alteration in the distribution and behaviour of parasites and their vectors [5]. This calls for controlled land use and other natural resources in order to minimize changes in the ecosystem. The recruitment of multidisciplinary experts is highly recommended to monitor and design the measures aimed at curbing the emergence or re-emergence of parasitic diseases [5].

The EIDs of wild animals have been placed into three groups viz: i) EIDs associated with spilt-over from domestic animals to wildlife populations living in proximity ii) EIDs related directly to human intervention, via host or parasite translocations and iii) EIDs with no overt human or domestic animal involvement [6]. One implication of these phenomena is that many wildlife species are reservoirs of pathogens that affect domestic animals and human health. The other implication is that wildlife EIDs pose a substantial threat to the conservation of global biodiversity.

The emergence of zoonotic and other parasitic diseases in particular usually occur in stages with an initial spill over event, followed by repeated small outbreaks in people, and the pathogen adaptation for human to human transmission. Both the emergence of new disease agents and the reemergence of disease pathogens that were initially phased out are a cause for concern to the society [7]. The array of infectious disease threats that require a redress is enormous, as a consequence, high rates that are currently challenging humanity are very high as a result of the high pace of emergence and re-emergence of pathogens worldwide. This has been attributed to the fact that the world has become a global village with regions being highly connected with resultant increase in business and humanitarian activities, hence there is a need to intensify the control of infectious agents at various regions to avoid further global spread [8]. Parasites can easily spread to different countries and continents of the world easily. For instance, they can be spread by moving animals and humans who are incubating such infections to the new destinations of the hosts. There are many possibilities to disseminate infectious parasites which make control efforts difficult. This is why it is absolutely necessary to develop and implement national, regional and global strategies for controlling infectious disease threats [8]. Increased interconnectivity and attendant ease of globalisation of food supply, increased international travel, increased population of susceptible individuals, change in eating habits, and improvement in diagnostic techniques have been attributed with the increased diagnosis of parasitic food-borne diseases [9].

The factors associated with emergence of infectious diseases are numerous and such as overpopulation, disruptions due to military actions, mass migration of populations due to national or man-made disasters, the migration of populations into large urban centres, and inadequate food and water supplies, environmental changes, human and animal demography, pathogen changes and changes in farming practice, social and cultural factors such as food habits and religious beliefs [10]. The exploitation of fallowed fields has resulted in the partial or permanent occupation of humans of such lands thereby sharing such locations with parasites and animals with the possibility of transmitting new infections [11]. Displacement of wildlife through development can result in both global warming [12] and epidemics of wildlife disease with the resultant spread of the infections to human populations.

Tourism has exposed the travellers to new infections initially limited to the hosts animals and humans and vice versa [13]. Chomel [14] has reported that emerging diseases are dominated by zoonoses and the majority of these diseases originate from wildlife. Most emerging parasitic infections are likely to be protozoans, however, some metazoans like Echinococus multilocularis are due to increased number of foxes in cities following the elimination of rabies in dogs in Europe [14]. The increased fox population in towns is likely due to the same reason of Toxocara canis infections [14].

Nigeria in particular and the entire West African region in general are not cut off/isolated from the threat of emerging and re-emerging animal parasitic diseases and other infectious diseases However, the rate at which such infections are discovered and reported would certainly be lower in the region compared to the general global pace perhaps due to poor surveillance, poor diagnostic methods and reporting amongst other reasons. For adequate documentation of the diseases, a number of factors should to operate not only in harmony but also regularly and frequently. All parties/components of the concerned team need to be informed of any new development and necessary on time action. This requires great deal of cooperation with the various governments and expert groups with the government assisting greatly in funding and in the enforcement of necessary regulations. The various governments in this region would certainly either due to lack 
of enough funds or lack of interest do not stress the need to carry out the necessary steps globally accepted in the monitoring and control of emerging infections. It is expected that the region being fertile with most of the factors that could lead to the proliferation of infectious diseases should also have a long list of such diseases. In this industrial age, developmental activities such as deforestation for building of homes, factories and roads to name but a few are equally common as found elsewhere, although the rate of the activities may be lower than that in developed nations. However, poor sanitation, the generally hot tropical climate, poverty, poor government prioritisation in disease control, climate change, floods, increased wars and civil conflicts with attendant displacement of large numbers of people and overcrowded refugee calms, malnutrition and misuse of drugs resulting in drug resistance are common and can seriously exacerbate the rate of disease development and spread.

The list of notifiable diseases (EIDs) in the Nigeria is expanding even though it may not be as long as those reported for some developed countries like America. This includes drug resistant malaria, African trypanosomosis, chikungunya, schistosomosis, babesiosis, cryptosporidiosis, Lyme disease, leishmaniiasis, filariasis, giardiasis, onchocerciasis, loiasis; yellow fever, dirofilariasis and hookworm (human helminthiasis).

\section{TYPES OF EMERGING AND RE-EMERGING ANIMAL PARASITIC DISEASES}

A good number of emerging and re-emerging diseases/parasites have been recognised and recorded in various parts of the world and are either helminth, protozoal/reickettsial or entomological/entomologicallyborne in origin. In Nigeria, some members of these classes of parasites are also common. These include: lyme boreliosis, cryptosporidiosis, malaria and yellow fever. However, many other emerging parasites and disease conditions which are not on this list but are also tagged as emerging and reemerging and occurring not necessarily only in Nigeria or the entire West African region but in other regions of the world have been listed under the relevant subheadings namely helminth, protozoa/rickettsial and entomological/ vector borne emerging and re-emerging animal parasitic diseases.

\subsection{Helminth Emerging and Re-emerging Animal Parasitic Diseases/Parasites}

Emerging and re-emerging helminth parasites are numerous and include Trichinella spp, Echinococcus spp, Onchocerca spp, Loa loa, schistosomosis, Aelirostrongylus abstrusus, Angiostrongylus vasorum, Angiostrongylus cantonensis, Baylisascaris cantonensis, Crenosoma vulpis, Dirofilaria immitis, Eucoleus aerophilis (Capillaria aerophila), Spirometra spp, Diphillobothrium spp, Gnathostoma spp, Opisthochis spp, Clonorchis sivensis, Umingmakstrongylus pallikuukensis, Protostrongylus stilesi, Teladosagia borearcticus, Setaria tundra, Taenia spp, and Cyclospora cayetanensis.

\subsection{Protozoal/Rickettsial Emerging and Re-Emerging} Animal Parasitic Diseases

This class of parasites is common and includes Erhlichiosis, Plasmodium knowlesi, Trypanosoma cruzi, Cryptosporidium parvuum, Giardia, Toxoplasma gondii, Leishmania, Sarcocystis, coccidiosis, tick toxicosis (tick paralysis), Lyme borreliosis (Lyme disease) and malaria. Ecological disturbances have been said to contribute immensely to the emergence and proliferation of malaria and zoonotic parasitic diseases including leishmaniasis, cryptosporidiosis, giardisis, trypanosomiasis, filariasis, onchocerciasis and loaisis [5]. It is has been established that each environmental change, whether occurring as a natural phenomenon or through human intervention, changes the ecological balance [5]. The most important food-borne parasites include the waterborne parasites transmitted by contaminated food such as Cyclosppora cayetanensis, Cryptosporidium, and Giardia [9].

Fasciolosis has become highly prevalent and exhibited by the increased numbers of Fasciola infections from less than 3 thousand to 17 million in the last decades [9]. Fasciolosis of humans and pigs are waterborne infections [9]. Faecal transmitted parasites are those transmitted through faecal or food contamination and include Toxoplasma gondii, and the emerging Trypanosoma cruzi and Echinococcus spp [9]. Malaria in humans is a disease caused by any one of four species of microscopic protozoan parasites in the genus Plasmodium and transmitted by mosquitoes in the genus Anopheles. Malaria was once common in the U.S. causing up to 4,000 deaths in 1935 but was later eradicated in 1950 . Malaria has bounced back in the U.S. with a wide prevalence [15].

\subsection{Entomological/Vector Borne Emerging and Re- Emerging Animal Parasitic Diseases}

This class of diseases/parasites is also greatly being recognised and includes

Sarcoptes scabei, tick toxicosis, tick paralysis and Lyme borreliosis (formerly known as Lyme disease), Chikungunya fever, dengue fever, yellow fever and plaque. Lyme disease is caused by the bacterium Borrelia burgdorferi which is transmitted between mammals by the black-legged tick, Ixodes scapularis. The most common early indication of Lyme disease in humans is a rash, followed by fever, flu-like symptoms, chills, headache and fatigue. Most cases of Lyme disease can be successfully treated with antibiotics $[15,16]$. Chikungunya fever is transmitted by aedes mosquito in man [3].

\section{CAUSES OF EMERGING AND RE-EMERGING ANIMAL PARASITIC DISEASES}

Emerging and re-emerging infections are on the rise because the predisposing/militating factors themselves are on the rise. Felissa et al. [17] have aptly described the causes as being both numerous and interrelated. It would not be too abstract to describe the developmental process as a sort of positive feedback mechanism, for example, globalisation has created global economic and social opportunities but at the 
same time it has led to emergence of diseases in some areas since humans, animals and goods are transported to target markets some times together with the pathogens they may be harbouring, thereby introducing such disease agents to the new destinations [15]. Greater interconnectivity means some disease agents initially localised in small areas will now be spread to new areas where they will be said to emerge from there. Some major causes of emergence and re-emergence of diseases have been listed [15] and these together with others have been outlined in section 3.1 to 3.11 .

\subsection{Climate Change/Global Warming}

There is a global ongoing climate change and some of its consequences such as the alteration in the habitats of parasites and other pathogens are obvious. The alteration in habitats has in turn resulted in the redistribution of infections. The implication is that there is a spread of parasites and other pathogens to areas which were initially too cold to harbour such organisms for instance. The infectious agents that have moved to occupy such new habitats are said to have emerged in such places and thus are termed emergent pathogens. By implication, the diseases usually caused by those pathogens will become common in hosts in the locations which were initially free or less affected [18]. Climate change which is itself an alteration in the climate could be a warming or a cooling effect. A warmer climate is more significant in this case as small increments in temperature have been shown to result in modifications of both the biotic and abiotic factors which in turn influence the activities of parasite vectors, parasites and their hosts in the environment. Generally, global warming enhances the development, multiplication and spread of many parasitic infections. Rising temperatures coupled with high stocking densities are a disadvantage to the hosts while an advantage to the parasites. This is because both the freeliving developmental stages of the parasites and their intermediate hosts/vectors develop and/or multiply faster at the higher optimum temperature [19-21]. For example, the rapidly growing numbers of muskox have led to high population densities which in turn has resulted in substantial environmental contamination with eggs and larvae of $T$. boreoarcticus, which may lead to increased exposure and transmission rates [22-25]. Vector-borne diseases are transmitted by insects such as mosquitoes, and arcarines such as ticks that are sensitive to temperature, humidity and/or rainfall. Temperature can also influence the reproduction and survival of an infectious agent within the vector, thereby further influencing disease transmission in areas where the vector is already present.

The protozoan disease known as malaria has increased tremendously in recent time and this increase is more serious in Africa due to insensitivity of the Plasmodium species to many anti-malarial drugs, the so-called drug resistant malaria. However, the disease is also being reported at cold placessuch as Kenyan plateaus. One of the most obvious causes apart from emergence of drug resistant species and movement of infected hosts to the areas is the rising temperatures. Rising temperatures are likely to encourage malaria to spread to many more locations which were initially free from the infection and increase the incidence of the infections in areas where it was initially low. The seasonal transmission and distribution of many diseases that are transmitted by mosquitoes (dengue, yellow fever), sandflies (leishmaniasis), and ticks (Lyme disease, tickborne encephalitis) which are usually more common in the hotter season may be made to spread to other seasons. It is feared that the rate of emerging infections could become very high in the temperate world since the warming climate will qualify the environment to become a habitat for pathogens which initially could not proliferate there [26].

\subsection{Changes in Biodiversity}

Another potential factor that greatly enhances the emergence of new pathogens is the proliferation of new strains of the pathogens. Different strains of parasites behave differently including their sensitivity to drugs and this is why the drug resistant strains of Plasmodium has led to the spread and increase prevalence of malaria. Numerous parasites have developed different strains with various potentials including those that can make them survive in harsh environments and rapid proliferation and down regulation of host immune responses.

\subsection{Population Mobility (Increased International Trade)}

Mass movement of people from their original locations and regrouping in new locations such as refugee camps as seen during wars and natural disasters like floods and droughts are very common now. This usually leads to over population in such locations and the consequence is always to create favourable conditions for new diseases to occur. Malnutrition, poor sanitation and breakdown in health have resulted to high rates of infections and diseases initially not common in some areas. When people migrate they do so with whatever pathogens they harbour and usually some disease agents spread from a few individuals to a multitude. Therefore, mass movements of people are extremely effective in carrying diseases to new areas. Increased international travel, commerce, and tourism provide efficient transport for disease-causing organisms and/or their vectors. They also greatly increase the exposure of people to "new" diseases for which they have little or no resistance.

\subsection{Movement of Animals}

Translocation of animals is necessary act because sometimes animals may need to be relocated to other areas for a number of reasons including feeding as seen in nomadism and transhumans, during droughts, marketing for slaughter and breeding and sought to safe grounds during war. Such movements although necessary are usually accompanied with some cost. No wonder, such movements have been blamed by some researchers to constitute a cause of emerging infections [27-31]. Fevre et al. [32] has also stated that the movement and co-occurrence of animals often result in transmission of parasites. For example, during drought and civil unrest as seen during wars cattle migrants to new save areas often introduce the parasites they harbour especially through the faeces of infected animals with gastrointestinal nematodes for example. In areas where the parasites were not found or were not common, such could then be termed emerging or re-emerging parasites to the new environment. Furthermore, the animals during grazing often 
share common pastures with other herds with a great possibility of each herd catching infections from each other.

\subsection{Globalisation of Commerce/Trade and Food Supply}

Commerce is much simplified these days due to the enhancement in various transport means. This has resulted in an increased transportation of animals, animal products and foodstuffs to areas where there is a huge demand for them. The movement of goods and humans serves as a driver for transporting various pathogens to the new regions, especially where boarder checks and screening of the good were not properly monitored. The transporters of the goods themselves and others also serve as a medium for transporting the pathogens from one location to the other.

\subsection{Social and Cultural Factors}

Methods of processing various foods vary from region to region and with culture and the habits of the people concerned. For example trichinosis although can be prevented by meat inspection, however, the best way of avoiding infections of such parasites is by proper cooking. This entails cooking for several minutes at temperatures of $100^{\circ} \mathrm{C}$ or higher. However, some consumers prefer "rare cooked" pork and such meat usually contains viable infective larvae of the parasites and consumers are likely to get infected. The prevalence rate of trichinosis is bound to be high in societies where the habit of eating "rare cooked" meat is common. Likewise, the incidence of cysticercosis is likely to be high among pork and beef consuming populations especially where poor sanitation, poor meat inspection and improper pork cooking are common because the chances of eating pork and beef with viable Cysticercus cellulosae and C. bovis. Hydatidosis will also be more common among sheep rearing populations.

\subsection{Trade of Infected Healthy Animals.}

This can facilitate the introduction and spread of disease. For example, infected animals when sold to distant places can likely introduce such diseases to the new areas especially if the climatic factors also are favourable for the infections to spread. This reiterates the need for quarantine services at strategic places such as boarders, sea and airports to help ascertain the status of animals before importing to different farms, different regions and to different countries.

\subsection{Reduction of Habitat}

Any environmental change, whether occurring as a natural phenomenon or consequent upon human manipulation would change the ecological balance, and context within which vectors and their parasites breed, develop, and transmit disease. The mix of vectors, their biodiversity, abundance, vector competence and human biting behaviour can be affected by changes occurring as a result of disruption of ecological stability. Deforestation and the resulting changes in land use, human settlement, commercial development, construction of roads, water control systems (dams, canals, irrigation systems, reservoirs), and climate have been accompanied by global increases in morbidity and mortality from a number of emergent parasitic diseases [5]. Among the factors that influence the burden of infectious diseases are environmental factors which change the distribution and impact of infections through many mechanisms [33]. For example, deforestation, with fragmentation of habitats, increases the edge effect, which then promotes pathogen-vector-host interactions; or some infectious diseases which may emerge or re-emerge as a result of a pathogen invading a niche newly created or recently vacated as a result of environmental change. Among the human-induced environmental changes that affect infectious disease risk are deforestation, road construction, irrigation, dam building, intensified crop and animal production systems, urban sprawl, poor sanitation, and pollution [33]. The clearing of new areas for the cultivation of food and other land uses resulted in human settlement in areas where animal populations and parasites were previously isolated from humans [7]. Extensive clearing of forest areas apart from contributing to global warming can also lead to displacement and stress of wildlife perhaps into new areas where they may either introduce or contract new infections with a possible spilt into human population in case of zoonotic parasites [12].

\subsection{Immunosuppressed Hosts}

An increase in hosts with suppressed immune systems increases the risk of infection to other diseases, including the EIDs. For example, some relationships have been found to exist in the incidence and severity of EIDs and immunosuppression.

\subsection{Host Density}

High host concentration per unit area of the habitat is a crucial factor that can modulate not only the spread of parasitic infections but it is also a potential cause of emerging parasitic infections $[23,24,34,35]$. This is because as the space available for the hosts is continuously occupied by the industrialization and other developmental projects, therefore animals of different breeds and species are bound to be pushed to cluster together on the same areas. Such overcrowded environments become greatly contaminated with loads of free-living developmental stages of parasites. As animals are pushed to new areas there is the likelihood for them to introduce their natural parasitic populations to the new environments which might never had such parasites; and such parasites become emergent parasites in the said areas causing and spreading emergent infections and diseases to the different breeds and species of animals in the habitats $[30,36,37]$.

\subsection{Improper Use of Drugs and Other Therapeutic Products}

Jorge [15] has also reviewed that the overuse and misuse of drugs have given rise to widespread resistance in the target organisms, thus making them more difficult to control diseases and/or their vectors.

The scenario of emerging and re-emerging animal parasitic diseases in Nigeria is quite similar to the global situation as emerging and re-emerging diseases are equally 
being reported in Nigeria in the same way as in other countries of the world, for example cryptosporidiosis which is emerging in Nigeria has also been reported as an emergent disease in the United States of America. Therefore, emerging and re-emerging infections are a global occurrence which is not surprising because some of the predisposing factors such as climate change, rapid infrastructural development and attendant loss of parasite habitat are unlimited and thus globally common. Nevertheless, as discussed earlier poor sanitation, poor awareness and poor prioritization by some governments may further aggravate the situation in the region.

The global response has been to curtail EIDs, minimise their occurrence and spread. The approach varies in different countries or regions but generally the emphasis is to improve the quality and sustainability of the control strategies such as surveillance, research/investigation and timely adoption of control/prevention measures, monitoring, improvement of infrastructure and skilled personnel. Many governments are being involved in the effort especially in terms of providing funds to run the control programmes and help the implementation of agreed policies.

\section{STRATEGY FOR EFFECTIVE MONITORING AND SURVEILLANCE OF EMERGING ANIMAL PARASITIC DISEASES IN NIGERIA}

There is a need to device and recommend practical and effective short and medium term strategies or measures, which if put in place would enable the Nigerian state to achieve the following goals: $i$. to have accurate data on the current status of emerging and re-emerging parasitic diseases in the country, ii. to effectively monitor and keep track of any changes, which may occur in future in the prevalence and epidemiology of this group of diseases, iii. to have an informed basis/data for national policy on the control of these parasitoses. For these goals to be achieved, the following strategies have been proposed:

a. Coordinated retrospective and prospective studies and prospective studies/surveys of prevalence and epidemiology of parasitic diseases/infections in domesticated animals and wildlife in different parts of the ecological zones of the country, as a source of baseline information. Such studies could be based on abattoir surveys, surveys of parasites of wildlife, studies of vector biology/ecology, analysis of records of diagnostic laboratories, Veterinary Teaching hospital clinics to name but a few.

b. Manpower/personnel training/development to produce knowledgeable 'resource persons' in the fields of modern Diagnostic Veterinary Parasitology and Entomology, Clinical/diagnostic Medicine, Veterinary Public Health, Epidemiology, Immunodiagnostics and applications of ICT in Veterinary Medicine.

c. Infrastructural development including well equipped diagnostic laboratories and modern abattoirs in designated centres in the major ecological zones of the country capable of undertaking accurate diagnosis of the major parasitic infections of domestic and wild animals. d. Well equipped Veterinary control posts, with quarantine facilities and effective links with laboratories in No " $\mathrm{c}$ " above, at our major sea, air and other international borders.

e. Enforceable legislation on restriction of imports of live animals and animal products as well as on reporting of unusual /uncommon, not necessarily 'notifiable' parasitic diseases to the Centre in No " $\mathrm{f}$ " below.

f. A national Coordinating and Reference Centre, for example, as part of existing setup at Vom, to handle and manage a national database for parasitic diseases and to disseminate on emerging/re-emerging parasites to all stake-holders at the Regional laboratories, Ministries, Vet Teaching Hospitals etc.

g. Co-operation with Veterinary and Animal Health authorities of neighbouring countries to ensure effectiveness of relevant, extant and new legislations.

i. Coordinated periodic, for example, 5-yearly, prospective studies in support of no "a" above.

There is already a base in Nigeria on how to contain emerging and re-emerging infections. What needs to be done is to build on that base being guided by the strategies for control of the infections which have been outlined above. Various procedures are being adopted in different regions over the globe. Although there may be many similarities, certain basic differences would definitely exist such as inclusion or exclusion of some procedures in order to enhance the control effort in the country. The sad thing however, is that most programmes in some developing countries are often characterised by ending up either with "big titles" or are poorly executed or lack continuity for a number a reasons. Factors like poverty, regime transitions, poor government prioritization, and corruption can result in the entrusting of important projects to the care of unqualified or inexperienced personnel and subsequent failure in the achievement of set goals of such programmes. This must be taken at heart in the application of the outlined strategies. Therefore training of personnel and recruitment of qualified and experienced professionals should be obligatory. Effecting of the strategies should be seen as a developmental programme and therefore given goals should be achieved over a specified period although with little room for modifications or inclusion of the unexpected.

It would take five years to fully implement the proposed strategies for effective control of emerging/re-emerging infections. New infections are likely to come up, therefore minds must be open so that there can be regular recruitment of experts with new ideas and experience or send local or existing experts/professionals for refresher courses or to attend conferences and workshops organised for hands-onexperience on new techniques for the diagnosis of new infections or infections recently reported in other areas in order to arm/equip them to monitor/survey and/or prevent such new happenings in the country. The activities should include: (i) Review of the existing installations including the audit of personnel and equipment and earmarking the deficiencies during the first year, (ii) In the second year, all deficiencies in both essential equipment and personnel should be corrected. Recruitment of the lacking staff, 
purchase of equipment and creation of enough accommodation for offices and laboratories should be accomplished. Training and /or retraining of personnel should continue. (iii) In the third year a review of the list of emergent/re-emergent infections should be made or reviewed comparing it with the list in the West African region and the entire global picture. Predisposing factors of the registered/diagnosed infections should be identified for control purpose. Reasons for any discrepancies in the number of emerging infections in the country and those in other places should be sought. (iv) Questions as to whether the differences were expected should be raised. Are the differences due to inability to diagnose properly or due to poor reporting and improper documentation? All these questions should be addressed in the fourth year and proper recommendations to amend the deficiencies should be made with immediate implementation. (v) In the fifth year, an assessment on the level of progress should be made and compared with the global standard and minimizing any differences that may arise. Thereafter regular checks and updating of staff and equipment and list of emerging infections should be emphasized. Working conditions should be made attractive such as giving incentives to staff in order to retain them as they acquire more and more experience.

\section{CONCLUSION}

The discovery of emerging and remerging parasitic diseases will definitely continue. This is because the infections have numerous possible origins and the predisposing factors are common and increasing. They are a threat to human and animal health and to the various economies. There is an absolute need for improved surveillance and monitoring to detect the factors that predispose to novel infections/diseases.

\section{CONFLICT OF INTEREST}

The authors confirm that this article content has no conflict of interest.

\section{ACKNOWLEDGEMENTS}

Declared none.

\section{REFERENCES}

[1] Lederberg J, Shope RE, Oaks SC, Jr., Emerging Infections. Microbial Threats to Health in the United States. Committee on Emerging Microbial Threats to Health, Division of Health Sciences Policy, Division of International Health, Institute of Medicine. National Academy Press, Washington, D.C. USA, 1992.

[2] World Health Organisation (WHO) (2004). Emerging zoonoses. http://www.who.int/zoonoses/emerging_zoonoses/en/ [Retrieved $2^{\text {nd }}$ June, 2014]

[3] Chhabra M, Mittal V, Bhattacharya D, Rana UVS, Lal S. Chikungunya fever: a re-emerging viral infection. Indian $\mathrm{J}$ Med Microbiol 2008; 26: 5-12.

[4] WHO (2014). Taeniasis/cysticercosis. Fact sheet No. 376. http://www.who.int/neglected_diseases/diseases/cysticercosis/en/ [Retrieved $3^{\text {rd }}$ June, 2014].

[5] Jonathan AP, Thaddeus KG, Nina G, Amy YV. Effects of environmental change on emerging parasitic diseases. Int $\mathrm{J}$ Parasitol 2000; 30: 1395-1405.
[6] Peter D, Andrew A, Alex DH. Emerging infectious diseases of wildlife: threats to biodiversity and human health. Sciences 2000; 287: 443-9.

[7] Penny H, Allison C, Megan VW, Thomas VI, Tara OT. Challenges to global surveillance and response to infectious diseases outbreaks of international importance. Biosecur Bioterror 2007; 5: No 3.

[8] Deirdre LC . Major factors affecting the emergence and reemergence of infectious diseases. Clin Lab Med 2004; 24: 559-86.

[9] Dorny P, Pract N, Deckers N, Gabbriel S. Emerging food-borne parasites. Vet Parasitol 2009; 3: 196-206.

[10] Louis MW. Zoonotic parasitic diseases: emerging issues and problems. Int J Parasitol 2008; 38: 1209-10.

[11] Chomel B, Bruno, Albino B, François-Xavier M. Wildlife, exotic pets and prevention of emerging parasitic zoonoses. Emerg Infect dis 2007 ; $13: 6-11$

[12] Hoberg EP, Polley L, Jenkins EJ, Kutz SJ, Veitch AM, Elkin BT. Integrated approaches and empirical models for investigation of parasitic diseases in northern wildlife. Emerg Infect Dis 2008; 14: 10-7.

[13] Centres for Disease Control and Prevention (CDC): Multistate outbreak of monkey pox-Illinois, Indiana, and Wisconsin, 2003. MMWR Morb Mortal Wkly Rep 2003; 52: 537-40.

[14] Chomel BB. Control and prevention of emerging parasitic zoonoses. Int J Parasotol 2008; 38: 1211-7.

[15] Jorge RR (2012). Emerging infectious diseases. University of Florida. University of florida. http://edis.ifas.ufl.edu/pdffiles/IN/ IN72200.pdf. Retrieved $3^{\text {rd }}$ June, 2012.

[16] Philippe P, Didier R (2001). Ticks and tickborne bacterial diseases in humans: emrging infectious threats. Clin Infect Dis 2001; 32: 897-928.

[17] Felissa RL.Factors contributing to the occurrence of emerging infectious diseases. Concept of Biol Res Nurs 2003; 4: 258-67.

[18] Kutz SP, Hoberg E, Polley L, Jenkins EJ. Global warming is changing the dynamics of Artic host-parasite systems. Proc R Soc 2005; 272: 2571-6.

[19] Kutz SJ, Hoberg EP, Polley L. A new lungworm in muskoxen: An exploration in Arctic Parasitology. Trends Parasitol 2001; 17: 27680 .

[20] Hoberg EP, Kutz SJ, Nagy J, et al. Protostrongylus stilesi (Nematoda: Protostrongylidae): Ecological isolation and putative host-switching between Dall's sheep and muskoxen in a contact zone. Comp Parasitol 2002; 691-9.

[21] Dobson A, Kutz S, Pascual M, Winfree R. Pathogens and parasites in a changing climate. In L. Hannah and T. Lovejoy (eds.), Climate change and biodiversity: synergistic impacts. Advances in applied biodiversity science 2003; 4: 33-38. Center for Applied Biodiversity Science, Conservation International Washington, DC.

[22] Grenfell BT. Parasitism and the dynamics of ungulate grazing systems. Am Nat 1992; 139: 907-29.

[23] Gulland FMD, Fox M. Epidemiology of nematode infections of Soay sheep (Ovis aries L.) on St. Kilda. Parasitology 1992; 105: 481-92.

[24] Arneberg P, Skorping A, Grenfell B, Read AF. Host densities as determinants of abundance in parasite communities. Proc R Soc London 1998, 265: 1282-9.

[25] Stien A, Irvine RJ, Lagvatn R, Albon SD, Halvorsen O. The population dynamics of Ostertagia gruehneri in reindeer: a model for the seasonal and intensity dependent variation in nematode fecundity. Int J Parasitol 2002; 32: 991-6.

[26] Dobson AP, Carper R. (1992). Global warming and potential changes in host-parasite and disease vector relationships. In: Peters 
RL, Lovejoy TE. Eds. New York: Yale Uni Press, 1995; pp. 20120.

[27] Williams ES, Yuill T, Artois M, Fischer J, Haigh SA. Emerging infectious diseases in wildlife. In: Bengis RG, Eds, Detection, diagnosis and management (Part 1). Paris France Office international des epizooties 2002; pp. 139-58.

[28] Daszak P, Cunningham AA, Hyatt AD. Emerging infectious diseases of wildlife threats to biodiversity and human health. Science 2000; 287: 443-9.

[29] Fayer R. Global change and emerging infectious diseases. J Parasitol 2000; 86: 1174-81.

[30] Deem SL, WB Karesh, W Weisman. Putting theory into practice: Wildlife health in conservation. Cons Biol 2001; 15: 1224-33.

[31] Harvell CD, Mitchell CE, Ward JR, et al. Climate warming and disease risks for terrestrial and marine biota. Science 2002; 296: 2158-62.
[32] Fèvre EM, Bronsvoort BMDC, Hamilton KA, Cleaveland S. Animal movements and the spread of infectious diseases. Trends Microbiol 2006; 14: 125-31.

[33] Wilson ME. Environmental change and infectious diseases. Ecosystem Health 2000; 6: 7-12.

[34] May, RM, Anderson RM. Regulation and stability for host-parasite population interactions II. Destabilizing processes. J An Ecol 1978; 47: $249-67$

[35] Dobson AP, May RM. Patterns of invasions by pathogens and parasites. In: Mooney HA, Drake JA, Eds. Ecology of biological invasions of North America and Hawaii. New York SpringerVerlag 1986; pp. 58-76.

[36] Lyles AM, Dobson AP. Infectious disease and intensive management: population dynamics, threatened hosts, and their parasites. J Zoo Wildl Med 1993; 24: 315-26.

[37] Dobson A, J Foufopoulos. Emerging infectious pathogens of wildlife. Phil Trans R Soc London 2001; 356: 1001-12.

(C) Atehmengo and Nnagbo; Licensee Bentham Open.

This is an open access article licensed under the terms of the Creative Commons Attribution Non-Commercial License (http://creativecommons.org/licenses/by-nc/3.0/) which permits unrestricted, non-commercial use, distribution and reproduction in any medium, provided the work is properly cited 